\title{
Abrupt Rotation of the Rashba Spin to the Direction Perpendicular to the Surface
}

\author{
Kazuyuki Sakamoto, Tatsuki Oda, Akio Kimura, Koji Miyamoto, Masahito Tsujikawa, \\ Ayako Imai, Nobuo Ueno, Hirofumi Namatame, Masaki Taniguchi, Johan Eriksson and \\ Roger Uhrberg
}

\section{Linköping University Post Print}

N.B.: When citing this work, cite the original article.

Original Publication:

Kazuyuki Sakamoto, Tatsuki Oda, Akio Kimura, Koji Miyamoto, Masahito Tsujikawa, Ayako Imai, Nobuo Ueno, Hirofumi Namatame, Masaki Taniguchi, Johan Eriksson and Roger Uhrberg, Abrupt Rotation of the Rashba Spin to the Direction Perpendicular to the Surface, 2009, Physical Review Letters, (102), 9, 096805.

http://dx.doi.org/10.1103/PhysRevLett.102.096805

Copyright: American Physical Society http://www.aps.org/

Postprint available at: Linköping University Electronic Press http://urn.kb.se/resolve?urn=urn:nbn:se:liu:diva-17497 


\title{
Abrupt Rotation of the Rashba Spin to the Direction Perpendicular to the Surface
}

\author{
Kazuyuki Sakamoto, ${ }^{1, *}$ Tatsuki Oda, ${ }^{2}$ Akio Kimura,${ }^{3}$ Koji Miyamoto, ${ }^{3}$ Masahito Tsujikawa, ${ }^{2}$ Ayako Imai, ${ }^{1}$ Nobuo Ueno, ${ }^{1}$ \\ Hirofumi Namatame, ${ }^{4}$ Masaki Taniguchi, ${ }^{3}$ P. E. J. Eriksson, ${ }^{5}$ and R. I. G. Uhrberg ${ }^{5}$ \\ ${ }^{1}$ Graduate School of Advanced Integration Science, Chiba University, Chiba 263-8522, Japan \\ ${ }^{2}$ Graduate School of Natural Science and Technology, Kanazawa University, Kanazawa 920-1192, Japan \\ ${ }^{3}$ Graduate School of Science, Hiroshima University, Higashi-Hiroshima 739-8526, Japan \\ ${ }^{4}$ Hiroshima Synchrotron Radiation Center, Hiroshima University, Higashi-Hiroshima 739-0046, Japan \\ ${ }^{5}$ Department of Physics, Chemistry and Biology, Linköping University, S-581 83 Linköping, Sweden
}

(Received 19 November 2008; published 3 March 2009)

\begin{abstract}
The polarization vector of the Rashba spin, which must be parallel to the two-dimensional (2D) plane in an ideal system, is found to change abruptly and definitely to the direction perpendicular to the surface at the $\bar{K}$ point of the Brillouin zone of a real hexagonal system, the Tl/Si(111)- $(1 \times 1)$ surface. This finding obtained experimentally by angle-resolved and spin-resolved photoemission measurements is fully confirmed by a first-principles theoretical calculation. We found that the abrupt rotation of the Rashba spin is simply understood by the 2D symmetry of the hexagonal structure.
\end{abstract}

The decrease in size of a crystal to nanometer scale leads to a reduction from a three-dimensional periodical structure to a lower-dimensional one that is often accompanied with the appearance of novel exotic solid-state properties [1-3]. In the case of a reduction into a two-dimensional (2D) system, a spin-polarized 2D electron gas (2DEG) that originates from the so-called Rashba-Bychkov (RB) (or simply Rashba) effect [4] is formed even for nonmagnetic materials. Generally, the electronic states of opposite spin orientation are considered to be degenerate in nonmagnetic materials, i.e., the Kramers degeneracy, due to the presence of both time-reversal and space-inversion symmetries. However, this degeneracy will be lifted by the spin-orbit interaction (SOI) when the latter symmetry is broken due to structural inversion asymmetry in a $2 \mathrm{D}$ system such as a crystal surface, and the SOI leads to a pair of split bands in the momentum $(\vec{k})$ space. In the case of an ideal 2DEG in the $(x, y)$ plane, the split originates from the RB Hamiltonian

$$
H_{\mathrm{RB}}=\alpha_{R}(|\epsilon|) \vec{\sigma} \cdot\left(\vec{k}_{\|} \times \hat{e}_{z}\right),
$$

where $\vec{k}_{\|}=\left(k_{x}, k_{y}, 0\right)$ is the in-plane momentum of a surface electron $\left(k_{\|}=\sqrt{k_{x}^{2}+k_{y}^{2}}\right), \hat{e}_{z}=(0,0,1), \alpha_{R}$ is the Rashba parameter $\left(\alpha_{R}=\hbar^{2} k_{0} / m^{*} ; m^{*}\right.$ is the effective mass and $k_{0}$ is the offset by which the $E\left(\vec{k}_{\|}\right)$parabola is shifted away from the $\bar{\Gamma}$ point), and $\epsilon$ is an electric field determined by the potential gradient normal to the surface. The split band is a completely polarized electronic state with the "spin polarization vector $(\vec{P})$ in the surface plane", and the directions of the $\vec{P}$ of the two bands are opposite, i.e., $\vec{P}\left(\vec{k}_{\|}\right)=-\vec{P}\left(-\vec{k}_{\|}\right)$.

This spin-splitting effect, which has been observed on clean surfaces of noble metals [5-10] and heavy group $V$ elements [11-13], has recently been reported to be en- hanced in systems in which heavy element atoms are adsorbed on light element substrates, such as $\mathrm{Bi}$ or $\mathrm{Pb}$ on a $\operatorname{Ag}(111)$ surface $[14,15]$. The spin-split bands observed in these studies show similar behaviors as those of the simple RB effect, i.e., they show pairs of split bands in the $\vec{k}$ space and the $\vec{P}$ almost lies in the surface plane. Up to now, all measurements on spin-splitting have been performed on metal and semimetal surfaces only, though the same phenomenon on semiconductor surfaces has a technological importance in spintronic devices like spin transistors $[16,17]$. A surface of a light element, such as $\mathrm{Si}$, with adsorbed $\mathrm{Tl}$ as the heavy element is a prime candidate for a semiconducting system with enhanced RB splitting. The adsorption of one monolayer (ML) of $\mathrm{Tl}$ on $\mathrm{Si}(111)$ leads to the $(1 \times 1)$ structure shown in Fig. 1(a) $[18,19]$.

In this Letter, we report the properties of the RB spin in a real $2 \mathrm{D}$ system, the $\mathrm{Tl} / \mathrm{Si}(111)-(1 \times 1)$ surface. We found that the surface-state band shows a "curious splitting" at
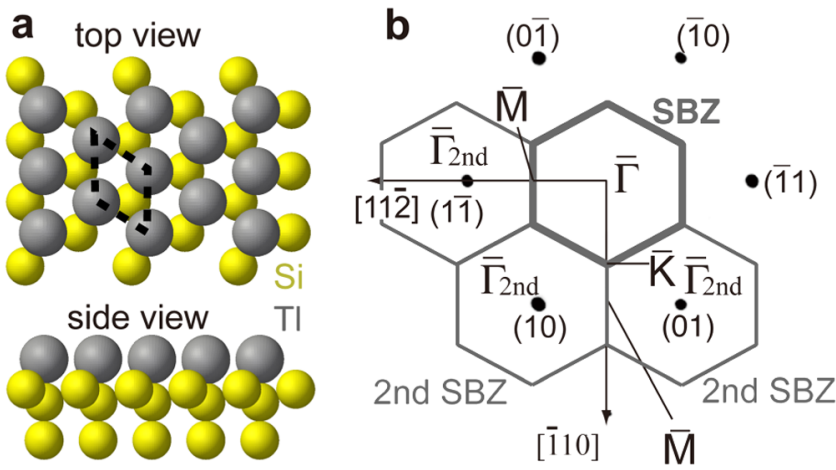

FIG. 1 (color online). (a) Schematic illustrations of the $\mathrm{Si}(111)-(1 \times 1)$ surface formed by the adsorption of $1 \mathrm{ML}$ of Tl. The thick dashed lines indicate the unit cell of this surface. (b) SBZs and LEED pattern of the $\mathrm{Tl} / \mathrm{Si}(111)-(1 \times 1)$ surface. $\bar{\Gamma}$, $\bar{M}$, and $\bar{K}$ are the symmetry points of the $(1 \times 1) \mathrm{SBZ}$. 
the $\bar{K}$ point of the surface Brillouin zone (SBZ), and that the $\vec{P}$ s of these spin-split bands point completely along the direction perpendicular to the surface at this symmetry point. These novel quantum phenomena, which have not been observed so far, are corroborated by the combination of high-resolution angle-resolved photoelectron spectroscopy (ARPES) and spin-resolved (SR)-ARPES measurements, and a state-of-the-art theoretical calculation. The abrupt rotation of the RB spin, which is simply understood by the $2 \mathrm{D}$ symmetry of the system, will not only add a new concept in spintronics, but also provide a deeper understanding to the physical properties of 2D materials, such as graphene. (Massless Dirac fermions have been observed with the Dirac point at the $\bar{K}$ point $[2,20,21]$ and the magnetic properties of graphene originating from the RB effect are reported to affect the electronic structure at $\bar{K}$ $[22,23]$.)

The ARPES measurements have been performed at beamline 33 of MAX-lab, Lund, Sweden, using synchrotron radiation light with a photon energy of $21.2 \mathrm{eV}$, and the SR-ARPES measurements have been performed in a spin-resolved photoemission system of the Hiroshima Synchrotron Radiation Center, Hiroshima University, Japan, using He I radiation $(21.22 \mathrm{eV})$ and a Mott detector operating at $25 \mathrm{keV}$. The energy resolution was approximately $50 \mathrm{meV}$ in the ARPES measurements and $200 \mathrm{meV}$ in the SR-ARPES ones, and the momentum resolutions were $0.06 \AA^{-1}$ and $0.02 \AA^{-1}$ at the $\bar{\Gamma}$ and $\bar{K}$ point, respectively, in both measurements. $\mathrm{Tl}$ was deposited on a $\operatorname{Si}(111)-(7 \times 7)$ clean surface, which was obtained by annealing at $1520 \mathrm{~K}$, from a Knudsen cell at a substrate temperature of $570 \mathrm{~K}$. The base pressure was below $4 \times$ $10^{-11}$ Torr during the measurements, and below $1 \times$ $10^{-10}$ Torr during the $\mathrm{Tl}$ evaporation. The effective Sherman function in the SR-ARPES measurement, $0.128 \pm 0.05$, was obtained by a self-calibration method in the maximum figure of merit $\left(1.8 \times 10^{-4}\right)$ [24].

A first-principles electronic structure calculation based on the Kohn-Sham theory [25], which employs ultrasoft pseudopotentials [26] and plane waves, was used in the theoretical study. This calculation has taken into account the fully relativistic effect that includes the SOI [27] and the self-consistent treatment [28] for two-componentspinor wave functions [29]. We used the generalized gradient approximation [30], the energy cutoffs of 25 and 300 Ry for wave functions and densities [31], and a repeated slab model, in which each slab contains a Tl monolayer (ML), $24 \mathrm{Si}$ ML and a H ML. Each slab was separated by a vacuum space of $9.7 \AA$. The atomic positions except those of the $\mathrm{H}$ atoms and $\mathrm{Si}$ atoms bonded to $\mathrm{H}$ were fully optimized to an assumed criterion of atomic force (less than $0.01 \mathrm{eV} / \AA$ ). In order to exclude the artificial dipole electric field imposed on the surface from the image cells in the repeated slab, the scheme of effective screening medium [32] was employed.

Figure 1(b) displays the SBZ of a $\mathrm{Tl} / \mathrm{Si}(111)-(1 \times 1)$ surface together with the low-energy electron diffraction (LEED) pattern of the sample shown by the black spots.

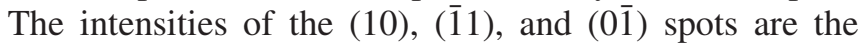
same, but the intensities of the (01), (10), and (11) spots are weaker than those of the three other spots. This indicates that instead of the sixfold symmetry of the 2D Tl layer, this surface has actually a three-fold symmetry resulting from the quasi-2D structure that includes the first Si layer.

The electronic band dispersion measured using ARPES along the $\bar{\Gamma}-\bar{M}-\bar{\Gamma}$ direction is shown in Fig. 2(a). Of the two observed bands, only the lower binding energy $\left(E_{B}\right)$ one is located in the gap of the projected bulk bands (hatched area [33]). This means that there is only one surface-state band on the $\mathrm{Tl} / \mathrm{Si}(111)-(1 \times 1)$ surface. Since the $6 s^{2}$ electrons of the $\mathrm{Tl}$ atoms act as an inert pair and are inactive in the Tl-Si bonding, which results in a monovalent character of the $\mathrm{Tl}$ atoms $[18,19]$, the observed surface-state band originates from the hybridization between the single $\mathrm{Tl}$ $6 p$ electron and one electron from the surface $\mathrm{Si}$ atom. Thus, there is no orbital degeneracy in the observed surface-state band. Further, no spin-split band is observable in Fig. 2(a), indicating that the Rashba energy $\left(E_{R}\right)$ of this system is small and cannot be resolved in the present ARPES study [the definition of $E_{R}$ and its value obtained in our theoretical calculation described below are shown in Fig. 4(b)]. Along the $\bar{\Gamma}-\bar{K}-\bar{M}$ direction, the surface-state
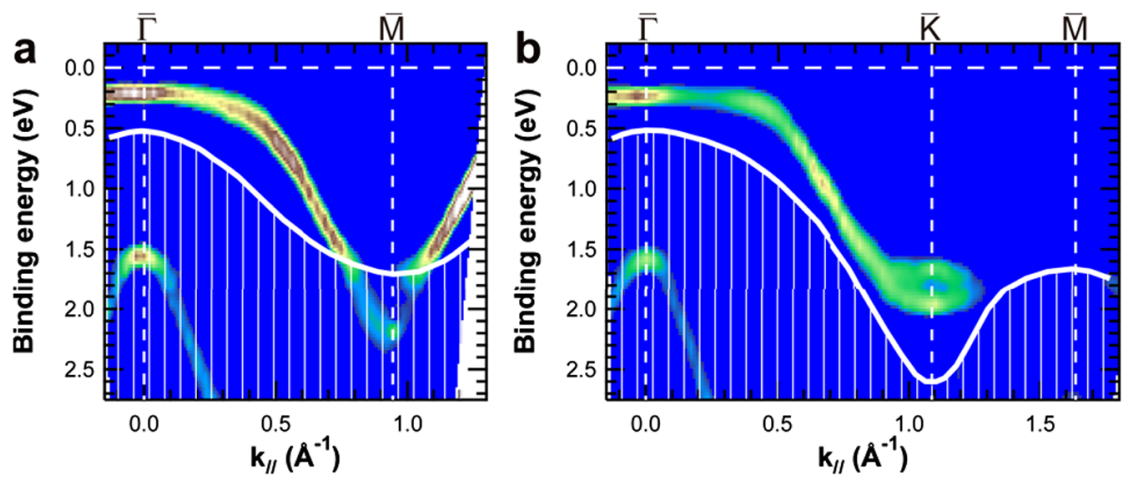

FIG. 2 (color online). Band structures measured along the (a) $\bar{\Gamma}-\bar{M}-\bar{\Gamma}$ and (b) $\bar{\Gamma}-\bar{K}-\bar{M}$ directions. The hatched areas show the bulk band projection taken from Ref. [33]. The horizontal dashed line at $E_{B}=0 \mathrm{eV}$ indicates the Fermi level. 
band shows a "curious splitting" around the $\bar{K}$ point that has not been observed on any other 2D or 3D system so far. Since there is no orbital degeneracy in the surface-state band, one of the most possible explanations is a spinsplitting. Note that the splitting appears along the energy axis at around $\bar{K}$ only, while if the split is due to a simple $\mathrm{RB}$ effect it would be observed in the whole SBZ except at high symmetry points such as $\bar{\Gamma}$.

Figure 3 shows the SR-ARPES spectra measured along the $\bar{\Gamma}-\bar{K}-\bar{M}$ direction with two different experimental setups that allow us to obtain separate spectra for different $\vec{P}$ in order to confirm the spin states of the electrons. In Fig. 3(a), the spin states with $\vec{P}$ that are parallel to the surface and perpendicular to the $\bar{\Gamma}-\bar{K}$ direction are degenerate at an emission angle $\left(\theta_{e}\right)$ of $0^{\circ}$, which corresponds to the $\bar{\Gamma}$ point. However, although this degeneracy agrees well with the ARPES results shown in Fig. 2, spin-split bands with in-plane spin polarization are observed at higher $\theta_{e}$. That is, the peak positions of the red and blue spectra are the same at $\theta_{e}=0^{\circ}$, but their $E_{B}$ become different at $\theta_{e}=$ $10^{\circ}$. This means that SR-ARPES further resolves the spin of the "single" band observed in ARPES. The small intensity of the higher $E_{B}$ peak (the red one for positive $\theta_{e}$ and blue one for negative $\theta_{e}$ ), which results from a final state effect for relativistic electrons [34], might hide the split feature in the spin-integrated ARPES spectra of Fig. 2.

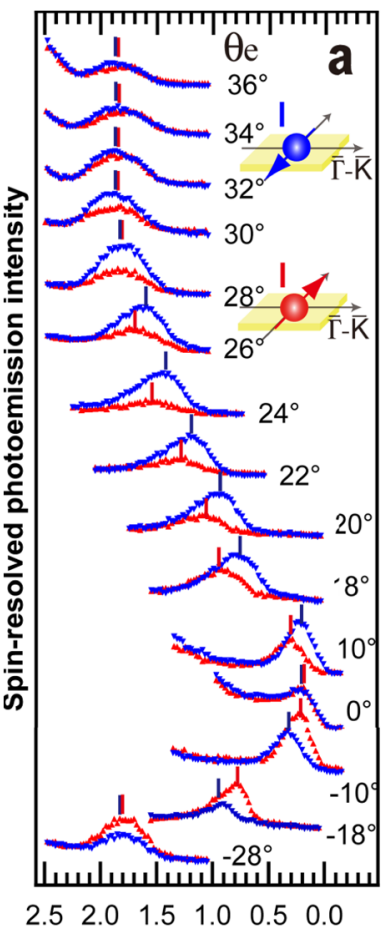

Binding energy (eV)

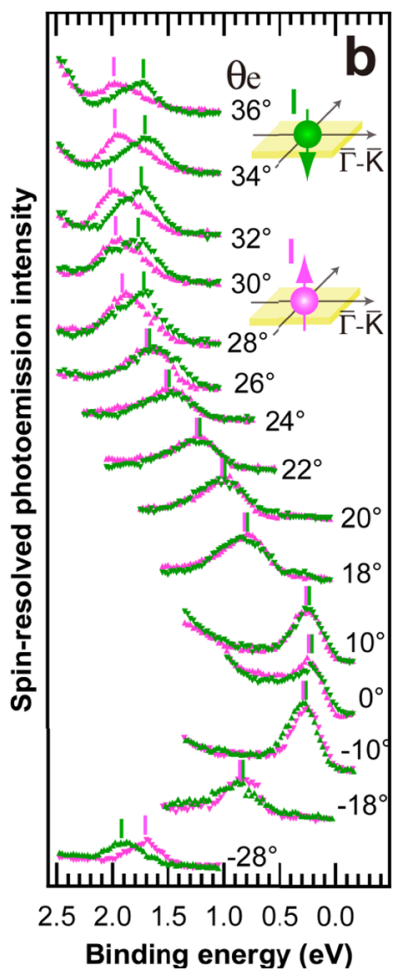

FIG. 3 (color online). (a) SR-ARPES spectra of the electrons with $\vec{P}$ parallel to the surface and perpendicular to the $\bar{\Gamma}-\bar{K}$ direction, and (b) spectra of the electrons with $\vec{P}$ perpendicular to the surface. $\theta_{e}=0^{\circ}$ corresponds to $\bar{\Gamma}$, and $\bar{K}$ is between $\theta_{e}=$ $32^{\circ}$ and $34^{\circ}$. The insets indicate the $\vec{P}$ of the detected electrons.
The spin-split bands are observable till $\theta_{e}=26^{\circ}$, but at $\theta_{e}=28^{\circ}$ and higher $\theta_{e}$, the difference in $E_{B}$ of the two bands becomes negligible.

The $\vec{P}$ perpendicular to the surface shows a completely different behavior. That is, the two spectra do not show any spin-splitting till $\theta_{e}=26^{\circ}$, but a clear split is observable at higher $\theta_{e}$. These results indicate that the $\vec{P}$ of the surfacestate band, which lies in the surface, "rotates abruptly" and points along the direction perpendicular to the surface around the $\bar{K}$ point $\left(\theta_{e} \sim 34^{\circ}\right)$. Note that in contrast to the $10 \%$ spin polarization aligned perpendicular to the surface plane, which results from the slight continuous rotation of $\vec{P}$ in the direction out from the surface plane on $\mathrm{Bi} / \mathrm{Ag}(111)$ and $\mathrm{Pb} / \mathrm{Ag}(111)$ surfaces [14,15], the spin polarization is $100 \%$ along the direction perpendicular to the surface around the $\bar{K}$ point only in the present case. In order to understand the origins of the abrupt rotation of the RB spin and the curious splitting around $\bar{K}$, that cannot be explained by the simple RB effect described in Eq. (1), we first discuss the RB effect in a real 2D system. Using Bloch wave functions $\varphi_{n \vec{k}}(\vec{r})=(1 / \sqrt{\Omega}) \exp (i \vec{k} \cdot \vec{r}) u_{n \vec{k}}(\vec{r})$, the effective SOI Hamiltonian of the "extended RB effect" can be described as

$$
H_{\mathrm{SOI}}(\vec{k})=\vec{\sigma} \cdot\left[\vec{\alpha}_{n}(\vec{k}) \times \vec{k}\right]+\vec{\sigma} \cdot \vec{B}_{n}(\vec{k}),
$$

where $\vec{\alpha}_{n}=\left(\hbar^{2} N / 4 m_{e}^{2} c^{2} \Omega\right) \int_{\text {cell }} d \vec{r}\left|u_{n \vec{k}}(\vec{r})\right|^{2} \vec{\nabla} V(\vec{r}) \quad$ and $\vec{B}_{n}(\vec{k}) \approx\left(\hbar^{2} N / 4 m_{e}^{2} c^{2} \Omega\right) \int_{\text {cell }} d \vec{r}(1 / r)(d V(\vec{r}) / d r) u_{n \vec{k}}^{*}(\vec{r}) \times$ $(\vec{\ell}) u_{n \vec{k}}(\vec{r})$. The second term of Eq. (2), which is not considered in the case of an ideal 2D system, is a Zeeman term that acts as an effective magnetic field to the band spin magnetization. The field $\vec{B}_{n}(\vec{k})$ is obtained as an averaged value on the angular momentum operator $(\vec{\ell})$, and is mainly caused by the atomic character of the wave function. When the wave function $u_{n \vec{k}}(\vec{r})$ is a good quantum state of the angular momentum operator, $\vec{B}_{n}(\vec{k})$ can have a large finite value and the electron spin tends to become parallel to the direction of $\vec{B}_{n}(\vec{k})$.

Figures 4(a) and 4(b) display the calculated spin-split band structure together with the experimental results. The good agreement between the dispersions of the calculated bands and the experimental ones corroborates the origin of the splitting at $\bar{K}$ to be spin-splitting. Further, the calculated band indicates that $k_{0}$ and $E_{R}$ are $0.2 \AA^{-1}$ and $20 \mathrm{meV}$, respectively. The direction of $\vec{P}$ of one of the split bands, as derived from the normal RB effect [Eq. (1) and the first term of Eq. (2)], is illustrated in Fig. 4(c). As shown in this figure, $\vec{P}$ rotates anticlockwise around $\bar{\Gamma}$ and clockwise around $\bar{M}$, whereas at $\bar{K}$, the rotations of the $\vec{P}$ along $\bar{\Gamma}-\bar{K}$ and those along $\bar{M}-\bar{K}$ are opposite. This opposite rotation implies that $\vec{P}$ of the normal RB spin is indeterminable at the $\bar{K}$ point. Indeed, the first term of Eq. (2) is found to vanish at $\bar{K}$ owing to its $C_{3}$ symmetry in the theoretical study. This means that only the second term contributes to the spin polarization at $\bar{K}$. The function 


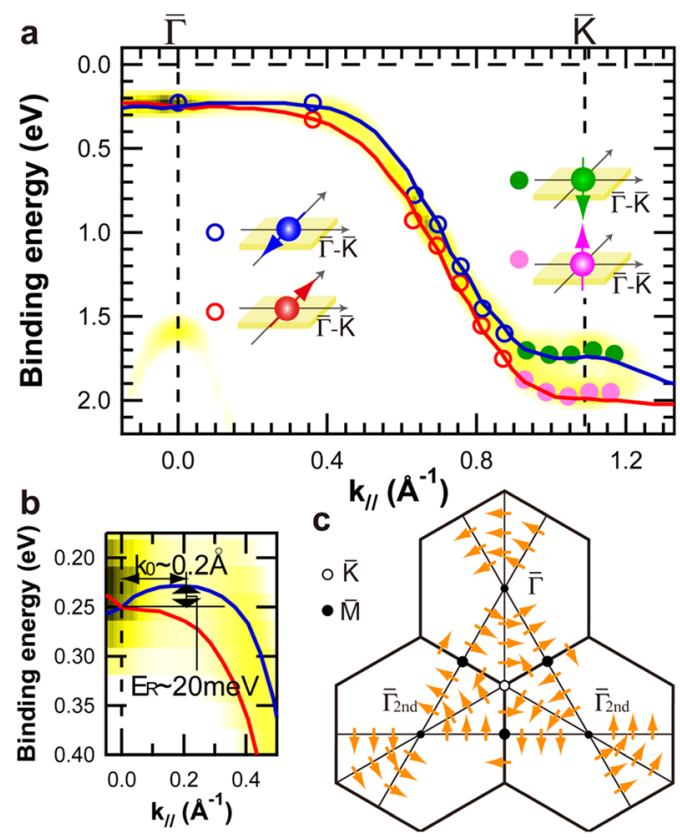

FIG. 4 (color online). (a) Spin-split surface band structures obtained experimentally (open and filled circles) and theoretically (solid lines) along the $\bar{\Gamma}-\bar{K}$ direction. (b) Band structures around the $\bar{\Gamma}$ point. $k_{0}=0.2 \AA^{-1}$ and $E_{R}=20 \mathrm{meV}$ are obtained from the calculated spin-split bands. (c) The arrows show the $\vec{P}$ of one of the split bands, as derived from the normal RB effect. The alternating direction of $\vec{P}$ around $\bar{K}$ is discussed in the text.

$p_{x}-i p_{y}\left(\right.$ or $\left.p_{x}+i p_{y}\right)$ forms an irreducible representation of the $C_{3}$ group when the SOI is neglected. When the SOI is on, the interaction lifts the spin degeneracy and, consequently, the effective field of $\vec{B}_{n}(\vec{k})$ becomes parallel to the $z$ direction, leading the spin polarization of the surfacestate band to be perpendicular to the surface. At the $\bar{\Gamma}$ point with $C_{3 v}$ symmetry and at the $\bar{M}$ point with $C_{1 h}$ symmetry, no spin-splitting has been observed neither experimentally nor theoretically. This is well explained by the fact that SOI cannot lift the spin degeneracy in these two symmetries.

In conclusion, our study reveals that a 2D system with hexagonal structure exhibits a novel quantum effect, the "abrupt rotation of the RB spin to the direction perpendicular to the surface", and that this effect is a general property of a reciprocal lattice point with $C_{3}$ symmetry. These results imply that the $\vec{P}$ of a real RB spin might be different from that of an ideal $\mathrm{RB}$ one depending on the symmetry of the sample. The present results will provide a deeper understanding on the physical properties of 2D materials, and they indicate that a proper knowledge of $\vec{P}$ and thus of the symmetry of the $2 \mathrm{D}$ band structure is indispensable to estimate the efficiency of the spin-current in a spintronic device. Moreover, the presence of rotated spins that are aligned with the surface normal suggests the possibility to double the functionality of spin-transistors by picking electrons with a certain wavelength or wave vector, i.e., one that corresponds to the $\bar{K}$ point.

Experimental support from T. Kadono, R. Nishimura, K. Kanomaru, and the MAX-lab staff is gratefully acknowledged. The calculation was carried out using the facilities of the Supercomputer Center, ISSP, University of Tokyo. This work was financially supported by the JSPS Grant-inAid for Scientific Research (A), 20244045, and the Swedish Research Council.

*Author to whom all correspondence should be addressed. kazuyuki_sakamoto@faculty.chiba-u.jp

[1] H. Ishii et al., Nature (London) 426, 540 (2003).

[2] Y. Zhang, Y.-W. Tan, H. L. Stormer, and P. Kim, Nature (London) 438, 201 (2005).

[3] D. Hsieh et al., Nature (London) 452, 970 (2008).

[4] Y. A. Bychkov and E. I. Rashba, JETP Lett. 39, 78 (1984).

[5] S. LaShell, B. A. McDougall, and E. Jensen, Phys. Rev. Lett. 77, 3419 (1996).

[6] G. Nicolay, F. Reinert, S. Hüfner, and P. Blaha, Phys. Rev. B 65, 033407 (2001).

[7] M. Hoesch et al., Phys. Rev. B 69, 241401(R) (2004).

[8] D. Popovic et al., Phys. Rev. B 72, 045419 (2005).

[9] H. Cercellier et al., Phys. Rev. B 73, 195413 (2006).

[10] D. Malterre et al., New J. Phys. 9, 391 (2007).

[11] Yu. M. Koroteev et al., Phys. Rev. Lett. 93, 046403 (2004).

[12] K. Sugawara et al., Phys. Rev. Lett. 96, 046411 (2006).

[13] T. Hirahara et al., Phys. Rev. Lett. 97, 146803 (2006).

[14] C. R. Ast et al., Phys. Rev. Lett. 98, 186807 (2007).

[15] F. Meier, H. Dil, J. Lobo-Checa, L. Patthey, and J. Osterwalder, Phys. Rev. B 77, 165431 (2008).

[16] S. Datta and B. Das, Appl. Phys. Lett. 56, 665 (1990).

[17] J. Nitta, T. Akazaki, H. Takayanagi, and T. Enoki, Phys. Rev. Lett. 78, 1335 (1997).

[18] S. S. Lee et al., Phys. Rev. B 66, 233312 (2002).

[19] K. Sakamoto et al., Phys. Rev. B 74, 075335 (2006).

[20] K. S. Novoselov et al., Nature (London) 438, 197 (2005).

[21] T. Ohta et al., Science 313, 951 (2006).

[22] Yu. S. Dedkov, M. Fonin, U. Rüdiger, and C. Laubschat, Phys. Rev. Lett. 100, 107602 (2008).

[23] A. Varykhalov et al., Phys. Rev. Lett. 101, 157601 (2008).

[24] K. Iori et al., Rev. Sci. Instrum. 77, 013101 (2006).

[25] P. Hohenberg and W. Kohn, Phys. Rev. 136, B864 (1964).

[26] D. Vanderbilt, Phys. Rev. B 41, 7892 (1990).

[27] T. Oda and A. Hosokawa, Phys. Rev. B 72, 224428 (2005).

[28] G. Theurich and N.A. Hill, Phys. Rev. B 64, 073106 (2001).

[29] T. Oda, A. Pasquarello, and R. Car, Phys. Rev. Lett. 80, 3622 (1998).

[30] J. P. Perdew et al., Phys. Rev. B 46, 6671 (1992).

[31] K. Laasonen, A. Pasquarello, R. Car, C. Lee, and D. Vanderbilt, Phys. Rev. B 47, 10142 (1993).

[32] M. Otani and O. Sugino, Phys. Rev. B 73, 115407 (2006).

[33] J. Ihm, M. L. Cohen, and J. R. Chelikowsky, Phys. Rev. B 22, 4610 (1980).

[34] J. Henk, M. Hoesch, J. Osterwalder, A. Ernst, and P. Bruno, J. Phys. Condens. Matter 16, 7581 (2004). 